\section{Factors associated with neuter status in \\ 12-month-old dogs in a longitudinal study}

\section{Rosa Da Costa ${ }^{1}$, Rachel Casey ${ }^{1}$, Joshua Woodward ${ }^{1}$, Rachel Kinsman ${ }^{1}$, Séverine Tasker ${ }^{2,3}$, Toby Knowles ${ }^{2}$, Michelle Lord ${ }^{4}$, Jane Murray ${ }^{1}$}

1 Dogs Trust, London, United Kingdom

2 University of Bristol, Bristol, United Kingdom

3 Linnaeus Group, Shirley, United Kingdom

4 Co-Evolve, Bristol, United Kingdom

\section{OBJECTIVES}

Neutering provides population control and health benefits for dogs. This study examined owner- and dogrelated factors associated with the reported neuter status of 12-month-old dogs in a longitudinal study.

\section{METHODS}

Online surveys were completed by UK/Republic of Ireland dog owners when puppies were aged $<16$ weeks and 12-months. Multivariable logistic regression analysis was used to examine associations between neuter status at 12-months and potential explanatory variables related to the owner (e.g. income, previous ownership and intention to work/breed dogs) and dog (e.g. sex, specific breed [yes/no], Kennel Club [KC] registration and source)

\section{RESULTS}

By age 12-months, 43.0\% (337/783) of dogs were reported as neutered. Dogs had lower odds of being neutered by 12 -months if registered with the $K C(O R=0.43,95 \% \mathrm{Cl}$ $0.31-0.59, \mathrm{P}<0.001)$ or if the owner intended to train them as sheep/cattle/gun dogs (OR $=0.42,95 \% \mathrm{Cl} 0.21$ $0.82, P=0.011$ ). Dogs acquired from charity/rehoming organisations had higher odds of being neutered by 12 -months $(O R=2.71,95 \% \mathrm{Cl} 1.25-5.88, \mathrm{P}=0.012) \mathrm{com}-$ pared with dogs that were homebred or acquired from KC assured, professional or hobby breeders. Intention at age $<16$-weeks to use the dog for breeding was associated with decreased odds of being neutered by 12 -months (OR $=0.13,95 \% \mathrm{Cl} 0.05-0.37, \mathrm{P}<0.001)$.

\section{STATEMENT (CONCLUSIONS)}

Whilst current UK veterinary guidelines recommend that veterinary advice on age of neutering should be sought on an individual basis, these results suggest that veterinary staff should consider raising awareness of neutering health benefits to owners of working dogs. Further research into neutering decisions relating to sheep, cattle and gun dogs is warranted.

\section{Epileptic seizures as unique neurological manifestation in a dog with methylmalonic aciduria without hypocobalaminemia}

\section{Despoina Douralidou, Alenka Hrovat, Beatrice Carletti}

Pride Veterinary Centre, Derby, United Kingdom

\section{OBJECTIVES}

To describe the case of a dog affected by methylmalonic aciduria (MMA) with normal serum cobalamin concentration and no signs of progressive encephalomyelopathy.

\section{METHODS}

A 1 year 9 months old female spayed Cockerpoo presented with a 5 months history of recurrent focal and generalised tonic-clonic seizures with a normal interictal behaviour. Investigations revealed hyperammonaemia, normal cobalaminemia and increased urinary methylmalonic acid excretion. Bile acid stimulation was normal and no changes compatible with portovascular anomaly were documented on abdominal ultrasound scan. The seizures resolved with temporary anticonvulsant (levetiracetam) therapy and by reducing the hyperammonaemia with lactulose. Trial supplementation with cobalamin alongside protein restricted diet were started. Seizures did not recur, and the dog remains neurologically stable 4 months following diagnosis.

\section{RESULTS}

MMA due to inborn error of metabolism resulting in cobalamin deficiency at a cellular level is suspected in this case. This can be caused by a failure of the cobalamin transport into the cells by the transcobalamin II protein, or a deficiency of the cellular methylmalonyl-CoA mutase enzyme, however these should be measured to confirm the exact pathophysiology.

\section{STATEMENT (CONCLUSIONS)}

Given that clinical signs of MMA are similar to those seen in dogs with portosystemic shunts and that commonly MMA is associated with cobalamin malabsorption and 